\title{
50. The Present Status of the Treatment of Head Injury in the Vicinity of Tokyo
}

\author{
Shigemaru Mıконсні \\ Hibiya Hospital \\ Chikao IzUmi \\ The National 2nd Tokyo Hospital \\ Hideyuki HiraI, Sigeo Toya \\ Keio University Hospital
}

133 cases required operative procedures out of the cases of the head trauma seen at Keio University Hospital, The National Second Tokyo Hospital and Hibiya Hospital from Jan. 1961 to Sept. 1964.

The 133 cases were consisted of 68 traumatic intracranial hematoma removed opeaatively and 65 other complications.

62 cases $(46.6 \%)$ among them were seen directly after the trauma at 3 above-mentioned hospitals and 49 cases $(36.8 \%)$ were referred to the specialist from local doctors for further medical care.

The rest (22 cases) were requested the consultation to the specialist in above 3 institutes not by the attending physician but by the patient's family mainly because of the distrust in the local doctor's treatment.

Accordingly, we staongly feel the urgent need to cultivate the local clinician to obtain the knowledge of the systematic appropriate first-aid for head trumatic patient, to establish the all-round practical training and educational system for the expert specialist and to initiate the campaign to build up the head injury centers.

\section{Diagnostic Value of LDH Isozyme Studies in Brain Tumors}

Hiroo Chigasaki, Kintomo TaKakura and Keiji Sano.

Depaatment of Neurosurgery, Faculty of Medicine, Univeriiy of Tokyo

The authors found that the levels of GOT (glutamic-oxalacetic transaminase), GPT (glutamic-pyruvic transaminase) and LDH (lactic dehydrogenase) in the serum and in the cerebrospinal fluid (CSF) were elevated in cases 
with malignant gliomas, whereas these values remained in normal range in bedign tumors. In individual cases, however, these values were so valiable that they were not reliable in diagnosis. This urged us to take a step forward to analyze the distribution of LDH isozymes in the CSF.

LDH isozymes were studied as described by VAN DER HELM. The CSF was dialyzed and condensed by hypertonic PVP. The sample was put on electrophoresis in agar gel and then the plate of the agar gel was incubated in a medium containing a dye of Nitro-BT. After incubation, the plate was washed with water and the proteins were fixed with acetic acid solution. The plate was subsequently dried and densitometry and planimetry were caraied out. Each fraction of $\mathrm{LDH}$ isozymes was named according to Wieme's method; namely from the anode to the cathode, $\mathrm{L}_{1}, \mathrm{~L}_{2}, \mathrm{~L}_{3}, \mathrm{~L}_{4}$ and $\mathrm{L}_{5}$.

The LDH isozyme distribution of the normal CSF was estimated as follows: $\mathrm{L}_{1}, 50 \% ; \mathrm{L}_{2}, 34 \% ; \mathrm{L}_{3}, 13 \% ; \mathrm{L}_{4}, 3 \%$ and $\mathrm{L}_{5}, 0 \%$. In patients with benign tumors such as meningioma, hemangioblastoma, acoustic neurinoma, teratoma and pituitary adenoma, the distribution of $\mathrm{LDH}$ isozymes in the CSF was quite different from that of LDH isolymes in the tissue homogenate of these brain tumors themselves. This fact that suggests that LDH in the CSF in these brain tumors does not derive from the tissues of the tumors. In cases of glioma, the distribution of $\mathrm{LDH}$ isozymes was characterized by the decrease of $L_{1}$ fraction and the relatively large elevation of $L_{4}$ and $L_{3}$ fractions, consequently showing the pattetn of $L_{2}>L_{1}>L_{3}>L_{4}>L_{5}$.

Since the tissue homogenate of gliomas showed a marked elevation of $L_{j}$ fraction in the CSF of the glioma seemed to derived from the tumor tissue itself. In a case of intracranial matastasis of liver cancer, the distribution of LDH isozymes in the CSF showed characteristic changes of the tumor tissue itself. In a case of parasitic granuloma, the distribution of $\mathrm{LDH}$ isozymes in the CSF resembled intimately that in cases of acute purulent meningitis. As cell counts in the CSF were very high in both conditions, LDH in the CSF might probably be attributed to the leakage from leucoxytes.

From these data, the authors believe that studies of LDH isozymes in the LDH isozymes in the CSF can be of diagnostic value in differentiating brain tumors. 